\title{
The relationship between eye position and egocentric visual direction
}

\author{
RAPHAEL BARBEITO and TREFFORD LEE SIMPSON \\ University of Houston, Houston, Texas
}

\begin{abstract}
Hering's model of egocentric visual direction assumes implicitly that the effect of eye position on direction is both linear and equal for the two eyes; these two assumptions were evaluated in the present experiment. Five subjects pointed (open-loop) to the apparent direction of a target seen under conditions in which the position of one eye was systematically varied while the position of the other eye was held constant. The data were analyzed through examination of the relationship between the variations in perceived egocentric direction and variations in expected egocentric direction based on the positions of the varying eye. The data revealed that the relationship between eye position and egocentric direction is indeed linear. Further, the data showed that, for some subjects, variations in the positions of the two eyes do not have equal effects on egocentric direction. Both the between-eye differences and the linear relationship may be understood in terms of individual differences in the location of the cyclopean eye, an unequal weighting of the positions of the eyes in the processing of egocentric direction, or some combination of these two factors.
\end{abstract}

A good framework within which to study headcentric, egocentric visual direction is a model usually attributed to Hering, but traceable to Wells a century earlier (see Ono, 1981). This model describes the essentials of egocentric direction, which include the retinal location stimulated (local sign) and the positions of the two eyes. To describe the phenomenology of egocentric direction, a point of origin from which directions are perceived also seems necessary and is included in the model. This point of origin is most often called the cyclopean eye. Together, these three aspects of the perception of headcentric, egocentric visual direction provide a good description of our perception of direction, as well as the basis of a model for egocentric directionalization (Ono, 1991; Park \& Shebilske, 1991; Swanston, Wade, \& Ono, 1990). This paper focuses on the eye position component of egocentric direction.

That the positions of both eyes affects egocentric visual direction is specified in the principles of visual direction extracted from Hering's writings (see Ono, 1979) and has been demonstrated to be true empirically even under conditions in which one of the two eyes is occluded (Ono \& Gonda, 1978; Ono \& Weber, 1981; Park \& Shebilske,

We thank H. E. Bedell, Hiroshi Ono, and two anonymous reviewers for their valuable comments on an earlier draft of this paper. Also, for technical assistance, we thank C. L. Kuether and Tong Ho, who were supported by Core Center Grant P30-EY07551 to the College of Optometry, University of Houston; Enita Torres for help in creating the graphics; and Becky Estrada for her ever-willing assistance in the library. This research was partially supported by a Research Initiation Grant to R. Barbeito from the University of Houston. Reprint requests should be sent to Raphael Barbeito, College of Optometry, University of Houston, Houston, TX 77204-6052.
1991). Although there appears to be little doubt of the importance of eye position to egocentric direction, the specific nature of this relationship has never been explicitly quantified. Although the implicit assumption of Hering's model is that the relationship is linear, at present there is only indirect evidence to suggest that this is true. In investigating the effect of curare-induced partial paresis, Matin et al. (1982) presented data from two subjects which indicated that each subject's errors in judgment of the subjective straightahead were linearly related to the presumed error in the extraretinal eye-position sense caused by the curare. Bridgeman and Fishman (1985) investigated whether a change in extraretinal eye-position information, induced by the attempt to manually rotate one eye, changes egocentric direction. Their data showed that the relationship between the error in localization of a monocularly fixated target and the amount of change in sensed eye position, as gauged by the secondary deviation in the occluded eye's position, was linear across the 10 individuals in their study. And, most recently, Park and Shebilske (1991) found that the relationship between phoria and egocentric direction within individual subjects is well fit by linear functions.

Thus, data suggest that the functional relationship between eye position and egocentric direction is linear, but these data are indirect. For example, Bridgeman and Fishman (1985), who found a linear relationship across subjects, did not investigate the nature of the relationship within subjects. And, whether open-loop (phoria) and closed-loop (fixation) variations in eye position are treated in the same way for egocentric direction is unknown. One of our purposes in the present study, then, was to specify the nature of the functional relationship between eye position and egocentric visual direction by quantifying it, 
thereby providing data that would allow a direct evaluation of the implicit assumption of Hering's model that the relationship is linear.

An additional and perhaps more important purpose of the present study was to address the tacit assumption of Hering's model that the position of both eyes affects egocentric direction equally. Data have been interpreted as indicating that the two eyes do not contribute equally to the processing of egocentric direction in one specific type of binocularly abnormal subject-constant strabismics with no prior history of clinical intervention (Mann, Hein, \& Diamond, 1979). In fact, Mann et al. concluded that, for the process of egocentric directionalization, the position of the constantly turned eye is completely ignored in this type of binocular abnormality. Furthermore, Walls (1951) believed that only one eye's position is processed for egocentric direction in binocularly normal individuals, and he made this difference in how each eye affects egocentric direction the basis of his theory of ocular dominance. Unfortunately, Walls (1951) provided no hard data in support of his proposition. But, although neither eye was ignored completely, Ono, Wilkinson, Muter, and Mitson (1972) did find between-eye differences in egocentric visual direction of a target associated with accommodative vergence in normals (a viewing situation proposed by Walls [1951] to demonstrate his theory). Ono et al., however, accounted for the between-eye differences that they observed in terms of the location of the cyclopean eye, not in terms of between-eye differences in sensed eye position.

In the present study, then, the effect of variation in the position of each eye on variation in egocentric direction was quantified, with the purpose of evaluating two implicit assumptions of Hering's model-namely, that the effects are (1) linear, and (2) the same for both eyes.

\section{METHOD}

\section{Apparatus and Stimuli}

The stimuli were two green light-emitting diodes, each mounted with an accommodative stimulus to aid in stable, accurate fixation. The stimulus for accommodation was a simulated Landolt $\mathrm{C}$ acuity target with a gap width of $.7 \mathrm{~mm}$, which at $50 \mathrm{~cm}$ corresponds to 5 ' of arc, or 20/100 in Snellen notation. One of the light-emitting diodes was used as the target stimulus for assessing egocentric direction. This target was positioned in the midline of the subject, $50 \mathrm{~cm}$ from the internodal axis. The other light-emitting diode was used to provide different eye-position configurations by acting as a binocular fixation stimulus. It was positioned at various distances between the subject and the target. This nearer fixation stimulus was always positioned to be collinear with one eye and the target, and to be slightly below it so as not to obscure the view of the target from the aligned eye. Since the two light-emitting diodes were collinear with one eye, different distances of the fixation stimulus lead to systematic variations in the positions of the nonaligned eye only. The light-emitting diodes were approximately at eye level.

In all, six eye-position configurations were used for each eye. Five were created by five distances of the fixation stimulusapproximately $16,20,24,30$, and $40 \mathrm{~cm}$ from the subject-and a sixth was created by binocular fixation of the target itself. Dis- tances of this fixation stimulus were chosen to provide approximately equal steps of convergence change between eye-position configurations with a total range of approximately 35 prism diopters. For the five configurations in which the nearer, light-emitting diode was a fixation stimulus, the fixation stimulus was continuously visible, and the target stimulus was flashed for $400 \mathrm{msec}$. For the sixth configuration, the target stimulus was continuously visible and the nearer light-emitting diode was extinguished.

To preclude the diplopia of the target that would occur with fixation on the nearer stimulus, the target was occluded from the nonaligned eye. Note, then, that local sign (the retinal area stimulated) was constant across stimulus configurations. Thus, any variation in egocentric direction of the target experienced by the subject over the stimulus configurations is attributable to variation in the position of the nonaligned eye.

During the experiment, the horizontal positions of both eyes were monitored with an infrared, photoelectric sensing system (Applied Science Laboratories, Eye Trak Model 210). This system was determined to be linear over a range of $\pm 15^{\circ}$ of arc, a range that was greater than the variation of eye positions used in the present study. Head position of the subject was restrained by a chin/headrest augmented by padding and straps to minimize head movements further.

Subjects were instructed to indicate the apparent direction of the target by pointing, with the index finger of the unseen hand, on a special surface. The pointing surface was plywood covered with Teledeltos paper, a paper made to have a uniform resistance across its entire surface (for details, see Bauer, Woods, \& Held, 1969). With a voltage applied across this paper, any lateral position on the surface is given, after suitable calibration, by the voltage at that position. A probe worn on the subject's index finger signaled the voltage, and therefore a pointing response, via appropriate wiring, to a microcomputer for processing.

The subject sat at a two-layered arrangement of table tops with the stimuli mounted on the upper layer. The lower layer, approximately $25 \mathrm{~cm}$ below the upper layer, not only was used to support the upper layer and to occlude the subject's arm while pointing, but also served as a rest for the subject's arm. The pointing surface was mounted perpendicularly under the upper table $50 \mathrm{~cm}$ from the subject, at the same distance as the target. This surface extended $40 \mathrm{~cm}$ on either side of the midline and $10 \mathrm{~cm}$ below the table top. The lower table was between waist and chest high, depending on the individual subject.

Both the analog information from the eye-position monitor and the voltages from the pointing surface were input to a microcomputer (Zenith 386) for on-line monitoring and recording. The data samples from each input channel were acquired via a 12-bit A/D converter board (SAC 12; QUA TECH, Akron, OH) every $40 \mathrm{msec}$ by software (CODAS; DATAQ Instruments, Akron, $\mathrm{OH}$ ) and stored to hard disk for subsequent analysis.

\section{Procedure}

After calibration of the eye-position monitor, the nearer stimulus was positioned collinearly with one eye and the target. The subject was instructed to fixate this stimulus and to signal the experimenter, by means of a pushbutton, when clear, single binocular vision was attained. The experimenter then flashed the target. Simultaneously with the flash, the positions of the two eyes were recorded by the microcomputer for subsequent validation of eye position. The subject then pointed (open-loop) to the apparent direction of the flashed target with the index finger of the preferred hand. After the pointing response was completed, the subject indicated to the experimenter, again by means of the pushbutton, that the pointing response was complete, and the experimenter stored the response on the hard disk. Each subject was instructed to rest his or her arm on the lower table after each pointing response. The subject was 
not required to maintain fixation on the stimulus between trials and usually used the between-trials interval to blink and look away, or to close his or her eyes.

Following this procedure, the pointing responses for a particular eye-position configuration were collected. The fixation stimulus was then moved to another distance and the procedure repeated, and so on for the five eye-position configurations requiring the nearer, fixation, stimulus. For the sixth eye-position configuration, the subject binocularly fixated the target and pointed in its apparent direction. In this case, as stated, the target was continuously visible. Subjects usually began with the nearest fixation distance and continued in sequence of diminishing convergence demand to the farthest fixation distance.

The general aim was to collect 10 directionalizations of the target (trials) per eye-position configuration. The actual number of trials varied, however, because of variation in the number of "clean" trials stored by the computer. In $90 \%$ of the cases, the actual number of trials used for data analysis was between 9 and 12 . In one case, the right-eye varying of Subject C.M., 20 trials per eyeposition configuration were collected and analyzed.

To ameliorate potential constant errors in the pointing responses resulting from our experimental paradigm-such as, for example, potentiation of the musculature of the arm used for pointing subjects were required to rest a few seconds after each pointing response. The duration of the actual pointing activity, approximately 3-4 sec, was usually briefer than the interval during which the arm was at rest.

After completion of the data collection for the six eye-position configurations, the subject took a break and the entire procedure was repeated for stimulus alignment to the other eye, or the subject returned on a subsequent day to complete the data collection.

The only light visible during the data collection and the pre- and postcalibrations of the eye position monitor was that from the lightemitting diodes.

\section{Subjects}

Five binocularly normal subjects participated in the complete experiment. All were students at the College of Optometry, but, with the exception of T.L.S., they were naive as to the purpose of the experiment. The experiment was conducted without the aid of optical correction. All subjects were isometropic. Also, all were emmetropic for the viewing distances used in the present study-except Subject D.W., who was 4.00 diopters myopic. For D.W., then, the target was defocused for all eye-position configurations, and the fixation stimulus was defocused for the three farthest distances. J.R. was the only left-handed subject. Phoria measurements for 4 subjects are provided in the Appendix.

\section{RESULTS}

For analysis, the mean apparent direction of the target under each of the six eye-position configurations for each eye was determined from the pointing responses. Each mean target direction was expressed as a deviation from the mean target direction obtained under binocular fixation. (Across subjects, the median standard deviation of the pointing responses was $0.64 \mathrm{~cm}$, with 25th and 75th percentiles of $0.50 \mathrm{~cm}$ and $0.84 \mathrm{~cm}$, respectively. Pointing responses were no more variable for the target when it was flashed than when it was continuously visible.)

Also, for analysis, an analogous set of predicted egocentric directions associated with variations in the position of each eye was derived by using Hering's model and the presumed positions of the eyes required for correct

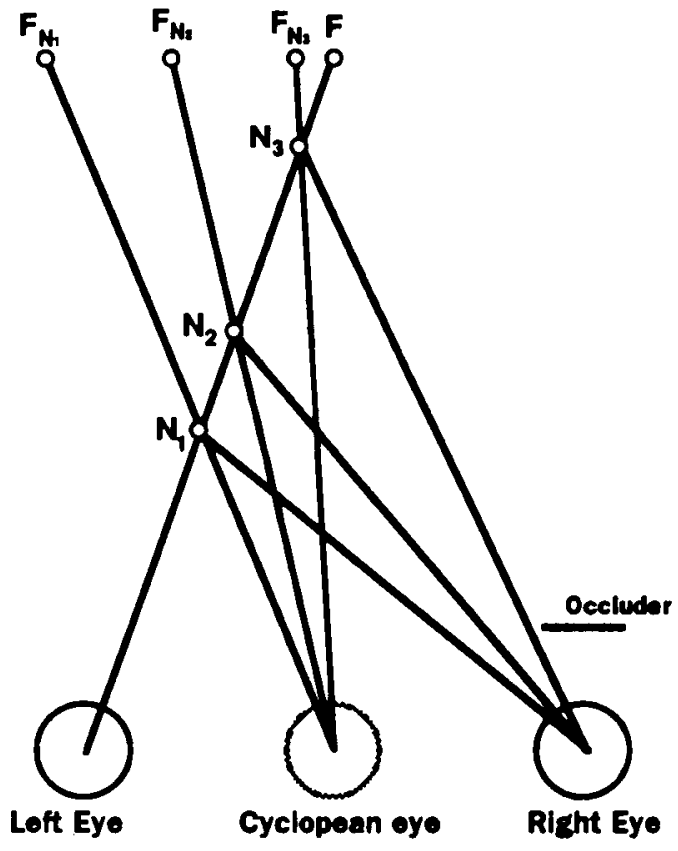

Figure 1. Schematic illustration of the stimulus arrangement and predicted directions of the target. Three of the distances of the fixation stimulus used to control eye position are indicted by N1, N2, and N3. The target to be directionalized, F, is predicted, according to Hering's principles, to be seen in the direction indicated by the line originating at the cyclopean eye and pasing through the fixation point. Here the expected egocentric directions of the target for fixation at N1, N2, and N3 are depicted as F1, F2, and F3. With binocular fixation, the tanget, $F$, is predicted to be correctly directionslized.

binocular fixation in each eye-position configuration. Figure 1 illustrates how these predictions were determined. Adjustments for eye position based on estimates of the actual eye position during target presentation (determined from the eye-position records) produced no meaningful differences from these predictions and were within the error for accuracy of the eye-position records.

The primary analysis of the data concerned the relationship between variations in the observed egocentric directions of the target stimulus and variations in the predicted directions associated with the different positions of the varying eye. Plots of the observed versus predicted egocentric directions for each eye of each subject are shown in Figure 2. Regression analysis confirms that the relationship between egocentric direction and eye position is linear. This linearity is evidenced by the substantial $r^{2}$ values for the least-squares regression fits, which range from 0.88 to 0.99 , with most being toward the higher end of this range. (For all but the binocular fixation condition, the target's predicted direction in the lefteye-varying condition, according to Hering's principles, is rightward of the target's veridical direction, and viceversa for the right-eye-varying condition. For simplicity, all perceived target directions on the predicted side of the 


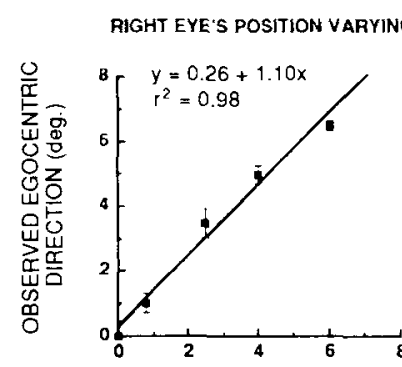

LEFT EYE'S POSTIION VARYING

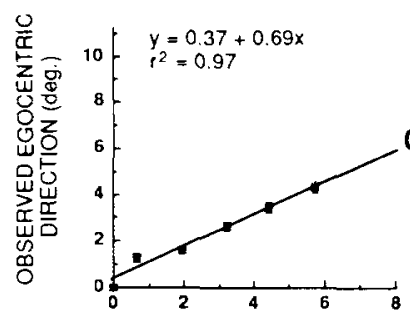

A.L.

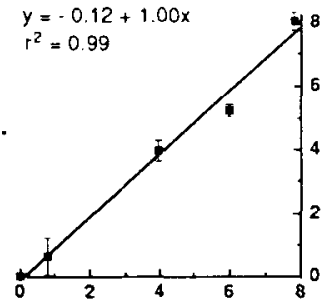

C.M.
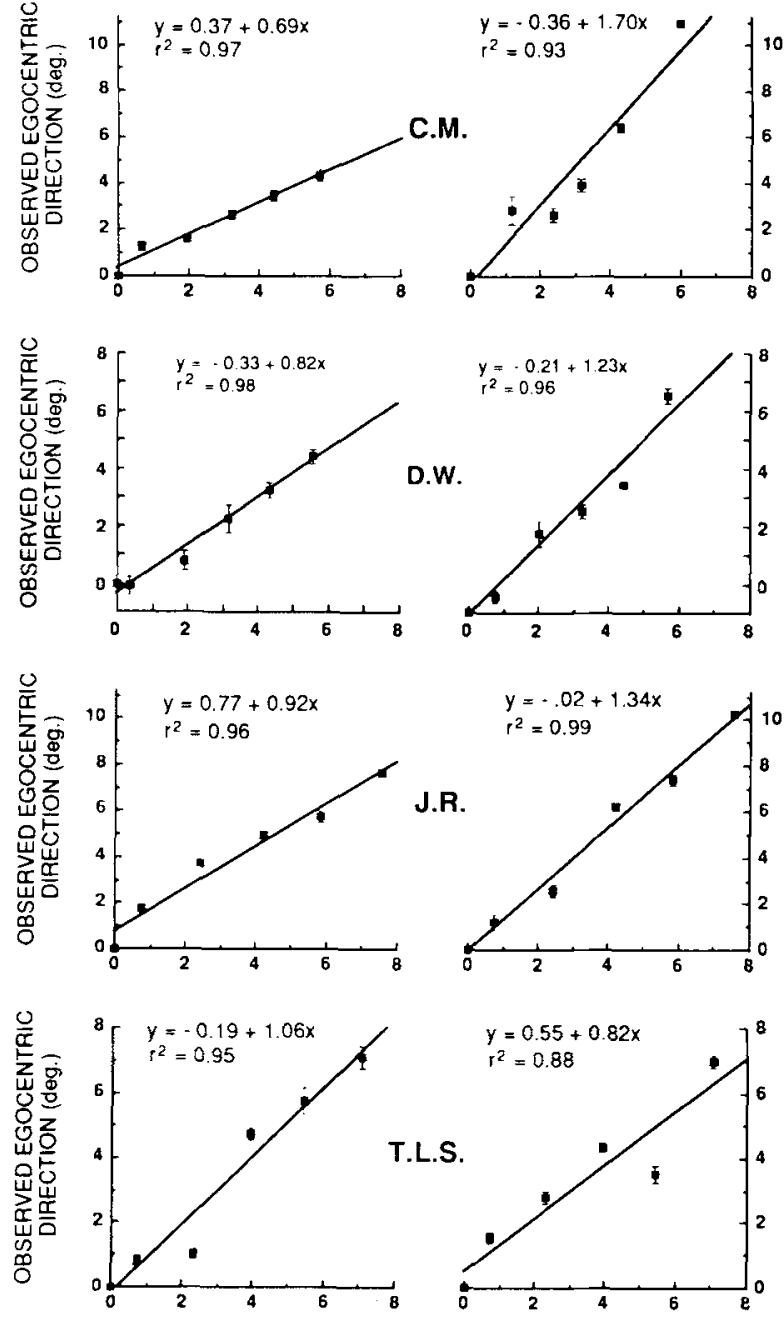

PREDICTED EGOCENTRIC DIRECTION (deg.)

Figure 2. The observed egocentric direction is plotted as a function of the predicted egocentric direction for the six eye positions examined in each eye of each subject. The line in each plot represents the least-squares regression fit. Note that the linear regression equations are also shown. For Subject A.L., data were collected for only five eye positions. Error bars represent \pm 1 standard error of the mean.

midline were given a positive sign. Thus, a negative sign indicates a target direction on the side of the midline opposite from that predicted.)

Further evidence of the linearity of the relationship is provided by consideration of the residuals of the linear regression analysis. Inspection of the residuals reveals no discernible pattern. Structureless residuals suggest that the variance in the observed egocentric direction which is not linearly associated with the variation in eye position is random error and is almost certainly due to the variable error of pointing.

A second, perhaps more notable, aspect of these linear functions is that the slopes of the best-fit lines for the two eyes of a subject appear to be unequal for some individuals. Between-eye differences in slope indicate that variation in one eye's position did not produce the same magnitude of change in egocentric direction as did variation in the other eye's position. Statistical analysis ( $F$ tests, using $\alpha=0.05$ ) of the slope parameter estimated in the linear regression analysis (see Table 1) indicated that greater-than-predicted variation in egocentric direction was associated with variation in the left eye's position for Subjects J.R. and C.M., and lesser variation than predicted for variation of the right eye's position for Subjects D.W. and C.M. (Because the subjects usually fixated the nearer fixation target position first and then continued in order of decreasing convergence demand, the possibility is raised that muscular effects such as posttetanic potentiation affected the results. However, the amount and duration of convergence in the present experiment was judged to be too short to produce these effects [Paap \& Ebenholtz, 1976]. And, more importantly, since Paap and Ebenholtz found no between-eye differences of potentiation, the between-eye differences found in the present study are not likely attributable to such an effect.)

For the analysis discussed thus far, the binocularly viewed target's predicted and observed directions were plotted at the $(0,0)$ point in each of the plots in Figure 2, and this point was included in the analysis. Use of the binocularly fixated target to "anchor" the plots in this way was justified because Hering's principles of visual direction predict that the target, when binocularly fixated, would be correctly directionalized. If this assumption is not tenable, what in the functions relating eye position and egocentric direction depicted in Figure 2 would differ? The data sets shown in Figure 2 were reanalyzed with linear regression analysis without this $(0,0)$ point. The results of this reanalysis-the slopes, intercepts, and $r^{2}$ values for each eye of each subject-are also shown in Table 1. Inspection of this table indicates that the goodness of fit of the regression lines was virtually unchanged by removal of this point from the plots. Furthermore, the Subjects J.R. and C.M. still exhibit between-eye differences in slope. The less-than-unity slope for the right-eyevarying case of $D$.W. was not sufficiently large to be significant statistically in this reanalysis. However, the direction of the deviation of slope from unity was the same in both analyses.

Without the constraint on the regression fits imposed by inclusion of the $(0,0)$ point, perhaps the biggest changes in the regression fits would be expected in the intercepts. In the original analysis, no intercepts were statistically different from 0 . In the reanalysis, although three of the 
Table 1

Results of Linear Regression Analysis on Data with and without Assumption of Correct Localization during Binocular Fixation

\begin{tabular}{|c|c|c|c|c|c|c|c|c|c|c|}
\hline \multirow[b]{2}{*}{ Subject } & \multicolumn{5}{|c|}{ Right-Eye Varying } & \multicolumn{5}{|c|}{ Left-Eye Varying } \\
\hline & Slope & $S E$ & Intercept & $S E$ & $r^{2}$ & Slope & $S E$ & Intercept & $S E$ & $r^{2}$ \\
\hline \multicolumn{11}{|c|}{ With Assumption } \\
\hline A.L. & 1.10 & 0.09 & 0.26 & 0.31 & 0.98 & 1.00 & 0.06 & -0.12 & 0.31 & 0.99 \\
\hline C.M. & $0.69^{*}$ & 0.06 & 0.37 & 0.20 & 0.97 & $1.70^{*}$ & 0.24 & -0.36 & 0.83 & 0.93 \\
\hline D.W. & $0.83 *$ & 0.06 & -0.33 & 0.19 & 0.98 & 1.23 & 0.13 & -0.21 & 0.43 & 0.96 \\
\hline J.R. & 0.92 & 0.09 & 0.77 & 0.40 & 0.96 & $1.34^{*}$ & 0.07 & -0.03 & 0.33 & 0.99 \\
\hline T.L.S & 1.06 & 0.12 & -0.19 & 0.50 & 0.95 & 0.82 & 0.15 & 0.55 & 0.63 & 0.88 \\
\hline \multicolumn{11}{|c|}{ Without Assumption } \\
\hline A.L. & 1.04 & 0.12 & 0.53 & 0.47 & 0.97 & 1.02 & 0.10 & -0.25 & 0.52 & 0.98 \\
\hline C.M. & $0.62^{*}$ & 0.05 & $0.69 \dagger$ & 0.17 & 0.98 & 1.79 & 0.35 & -0.74 & 1.34 & 0.90 \\
\hline D.W. & 0.89 & 0.05 & $-0.58 \dagger$ & 0.18 & 0.99 & 1.27 & 0.18 & -0.40 & 0.65 & 0.94 \\
\hline J.R. & 0.81 & 0.07 & $1.38 \dagger$ & 0.31 & 0.98 & $1.34^{*}$ & 0.11 & -0.05 & 0.51 & 0.98 \\
\hline T.L.S. & 1.09 & 0.17 & -0.35 & 0.76 & 0.93 & 0.73 & 0.20 & 0.98 & 0.90 & 0.82 \\
\hline
\end{tabular}

Note-The $F$ tests for the slope and intercept parameters had $d f=(1,4)$ in the with-assumption case, and $d f=(1,3)$ in the without-assumption case. *Statistically different $(p<.05)$ from a slope of 1 . TStatistically different $(p<.05)$ from an intercept of 0 .

five intercepts were statistically different from 0 for the right-eye-varying condition, none was significant for the left-eye-varying condition. Thus, the data as a whole do not clearly permit rejection of the assumption that the binocularly viewed stimulus is correctly localized.

A final question to be considered is how well variations in the pointing responses represent variations in the perceived egocentric directions. Although investigations of egocentric visual direction often measure perceived direction with pointing responses or some variant thereof (e.g., Barbeito \& Ono, 1979; Bedell, Klopfenstein, \& Yuan, 1989; Gauthier, Nommay, \& Vercher, 1990; Steinbach \& Smith, 1981), individual differences in perceived egocentric direction are also found with visually based measures, as in the adjustment of the position of a visual target to match the remembered apparent direction(s) of a target (or targets) (Barbeito, 1981; Bedell et al., 1989; Ono \& Barbeito, 1982; Park \& Shebilske, 1991). In the present study, qualitative verbal reports from Subjects C.M. and D.W. about their visual impressions corroborated their pointing data, in that larger variation in egocentric direction was associated with the left-eye-varying condition than with the right-eye-varying condition. An additional point in this vein is that differences in magnitude of variation between targets perceived rightward of the midline (left eye's position varying) and leftward of the midline (right eye's position varying) are not reasonably attributable to the fact that only either the right or the left arm was used for pointing, for the following reasons. First, Subjects C.M. and J.R. provided larger-thanexpected variations in egocentric direction (slopes statistically greater than unity) for targets perceived rightward of the midline even though the left hand was used by Subject J.R. and the right hand by Subject C.M. And second, an informal control experiment, in which the 2 subjects (C.M. and D.W.) who showed smaller-than-expected variations in the right-eye-varying condition participated, showed that these variations are probably not attributable to "under-pointing"' simply because the target was per- ceived left of the midline. These 2 right-handed subjects open-loop pointed (9-11 trials) to a binocularly fixated target presented either in the midline or $5^{\circ}$ on either side of it at $50 \mathrm{~cm}$. With the mean direction of the midline target as a " 0 " " point, the means (and standard errors) were as follows: for Subject C.M., left of midline, $5.46^{\circ}$ $\left(0.29^{\circ}\right)$, right of midline, $7.89^{\circ}\left(0.41^{\circ}\right)$; for Subject D.W., left of midline, $5.77^{\circ}\left(0.19^{\circ}\right)$, right of midline, $4.46^{\circ}\left(0.34^{\circ}\right)$. Note that most of the predicted target directions were within $5^{\circ}$ of the midline.

\section{DISCUSSION}

The linear relationship between eye position and egocentric visual direction found in the present study provides empirical substantiation for the implicit assumption of linearity found in Hering's model and thus extends the findings of previous studies (Bridgeman \& Fishman, 1985; Matin et al., 1982; Park \& Shebilske, 1991). However, the results indicate that the magnitudes of changes in egocentric direction associated with variation in one eye's position are not the same as changes associated with variation in the other eye's position in all subjects. This finding is not consistent with strict expectations based on Hering's model.

A question now arises: To what can these between-eye differences be attributed? At least two possible factors could, in principle, lead to between-eye differences in perceived egocentric direction. One is that the location of the cyclopean eye is noncentral, and the other is that each eye's position carries a different weight for the determination of egocentric direction.

\section{A Noncentral Cyclopean Eye?}

In Hering's model, the position of the cyclopean eye is assumed to be midway between the eyes, a location that, other things being equal, would lead to the variations in egocentric direction associated with each eye being equal in our experimental paradigm. According to other ele- 


\section{BETWEEN-EYE DIFFERENCES IN SLOPE: NON-CENTRAL CYCLOPEAN EYE?}
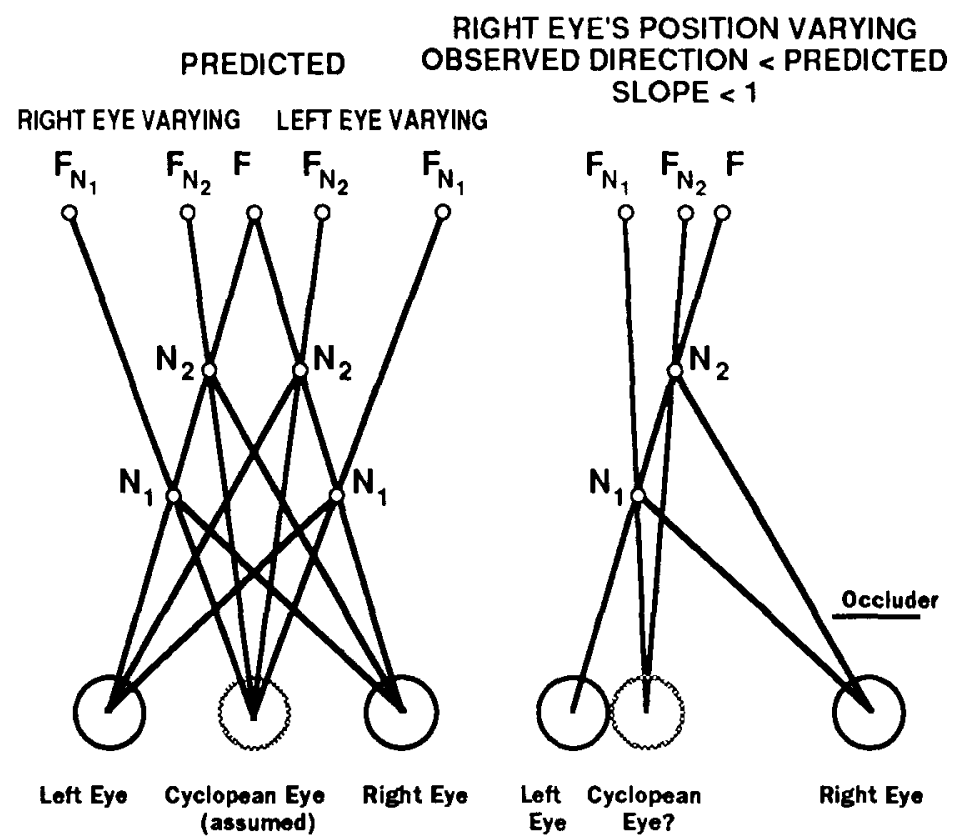

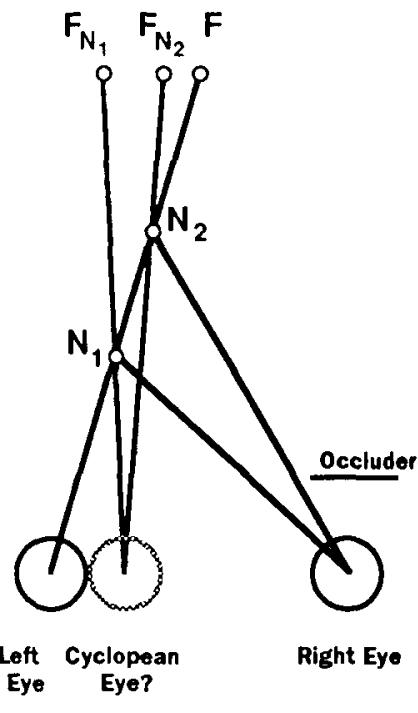

\author{
LEFT EYE'S POSITION VARYING \\ OBSERVED DIRECTION > PREDICTED \\ SLOPE $>1$
}

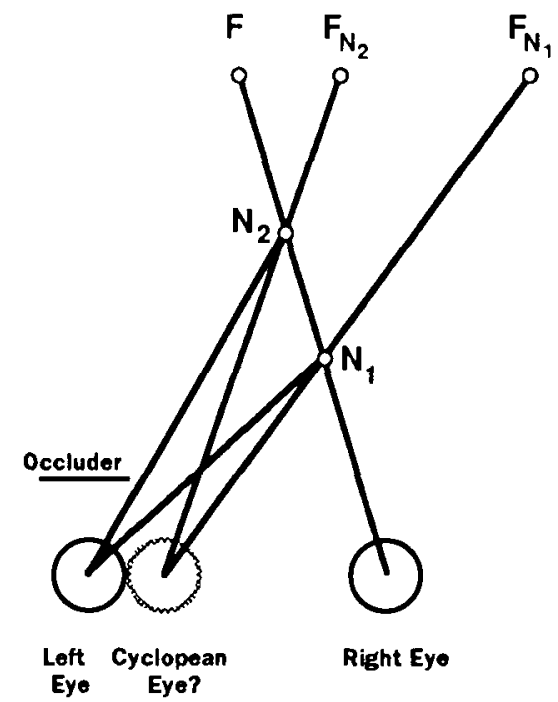

Figure 3. A cyclopean eye deviating from the midline toward the left eye is shown (central and rightmost portions) in order to illustrate how a noncentral cyclopean eye could account for between-eye differences in the experimental paradigm used. Following the procedure for determining the predicted directions of the target, F, illustrated in Figure 1, a subject with a left-of-center cyclopean eye would perceive a smaller than predicted variation in egocentric direction with the right eye varying and a correspondingly larger than predicted variation with the left eye varying. The leftmost portion of the figure illustrates, simultaneousiy, the equal between-eye variations in egocentric direction predicted with a central cyclopean eye.

ments of Hering's model, however, a noncentral cyclopean eye would lead to the expectation of unequal variations in egocentric direction. For example, as can be seen in Figure 3, a cyclopean eye on the internodal axis but closer to the left eye would lead to larger variations in perceived egocentric direction associated with variation in the left eye's position and correspondingly smaller variations in direction with variation in the right eye's position. Since the predicted variations ( $x$-axis) used for the plots in Figure 2 were based on a central cyclopean eye, observed egocentric directions ( $y$-axis) associated with a left-of-center cyclopean eye in this hypothetical case would show up as a slope greater than unity for the lefteye-varying situation and less than unity for the right-eyevarying situation. In fact, between-eye differences in egocentric direction of the sort reported here have been reported previously and attributed to a noncentral cyclopean eye (Ono et al., 1972).

In principle, then, a cyclopean-eye location could be estimated for a subject that would produce predicted egocentric directions in agreement with those observed in the present study and so account for the between-eye differences. When the observed egocentric directions are plotted with these new predictions, a slope of 1 for each eye-varying condition would, within the limits of measurement error, result. Such locations were estimated with the criterion that the quality of the regression fits, as measured by the $r^{2}$ values, not be meaningfully affected. As an example, these new regression fits are shown in Figure 4 for Subject C.M., who showed the most extreme between-eye differences. To fit his observed set of egocentric directions, a cyclopean eye $1.1 \mathrm{~cm}$ toward the left eye from the midline and $2.5 \mathrm{~cm}$ in front of the internodal axis was necessary. This cyclopean-eye location produces predicted variations in egocentric direction almost identical to those observed in both eye-varying conditions. Also, this location is within the range of cyclopean eye locations found when the cyclopean eye is measured directly (see, e.g., Barbeito, 1981; Barbeito \& Ono, 1979).

\section{A Between-Eyes Difference in Weight?}

The notion of a noncentral cyclopean eye has been invoked in previous investigations to account for individual differences in the perception of egocentric direction (e.g., Barbeito, 1981; Ono \& Barbeito, 1982; Ono et al., 1972). But, these studies, along with studies that formally measured the location of the cyclopean eye by measuring subjects' directionalizations of visual targets (e.g., Barbeito $\&$ Ono, 1979), assume implicitly that the two eyes are given equal weight for egocentric direction. To what extent reported individual differences in egocentric direc- 
RIGHT EYE'S POSITION VARYING

LEFT EYE'S POSITION VARYING
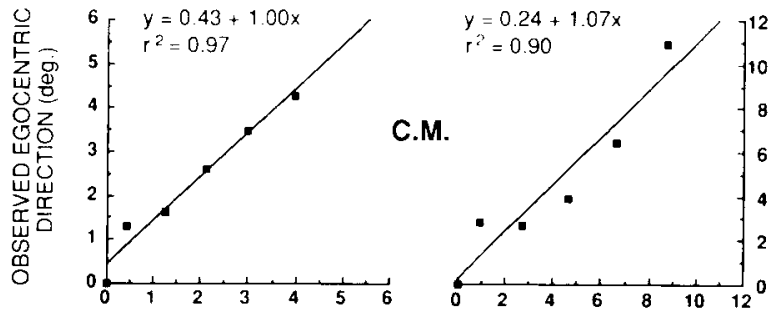

PREDICTED EGOCENTRIC DIRECTION (deg.) BASED ON NON-CENTRAL CYCLOPEAN EYE

Figure 4. The observed egocentric direction is plotted as a function of the predicted egocentric direction based on a cyclopean eye $1.1 \mathrm{~cm}$ toward the left eye from the subject's midline and $2.5 \mathrm{~cm}$ in front of the internodal axis.

tion could also be attributed to individual differences in how eye position is weighted was not addressed by these studies. Evidence from several sources taken together supports the possibility that the eyes might carry different weights for egocentric direction. For the purposes of exposition, this evidence may be separated into two categories, one relating to oculomotor aspects and the other to sensory aspects.

An oculomotor aspect? Morgan (1978) reported errors in egocentric direction attributable to misrepresentation in eye position. The amount of error depended on the amount of rotation of the eye from the primary direction of gaze; greater rotation led to greater error in directionalization and, by implication, to greater misrepresentation of eye position. Similar results were reported by Hill (1972), but neither study investigated the possibility of between-eye differences in the misrepresentations they reported. However, the results of Mann et al. (1979) have been interpreted as addressing this question. They investigated binocularly abnormal subjects-namely, strabismics-and reported that there can be very large between-eye differences in the extent to which the position of the occluded eye affects the egocentric direction of a monocularly viewed target. And evidence from the oculomotor literature supports the possibility of betweeneye differences. Viirre, Cadera, and Vilis (1987) found, in monkeys (macaca fascicularis), that after 1 week of occlusion, the amplitude of the saccades in the occluded eye that accompany the saccades of the viewing eye changed. They concluded that different commands to motoneurons can be sent to one muscle of a conjugate pair without affecting commands to the other. The possibility that the two eyes can be driven independently and unequally raises the possibility that the two eyes can simultaneously carry different eye-position information for egocentric direction.

Further analysis of the data of the present study, however, does not clearly support the existence of betweeneye differences in the representation of eye position for egocentric direction. Within the framework of Hering's model, one clear prediction of between-eye differences in weight would be that the direction of the target under binocular viewing would be nonveridical, regardless of the cyclopean eye's actual location. In the present study, this expectancy would be most evident in the regression analysis that excluded the $(0,0)$ point (see the "without assumption" section of Table 1), and it should be evidenced as nonzero intercepts of the best-fit regression lines. However, for the subjects with regression-line slopes most clearly greater or less than the expected slope of 1 , the zero intercept (no eye-position error) usually fell within the limits of the intercept's statistical error. And when there is a statistical difference from 0 , the difference is not always in the direction necessary to explain the between-eyes difference in terms of differential weight. (The more direct comparison of the perceived direction of the target when it was binocularly fixated with its veridical direction was not used, because the perceived direction would potentially be more affected by constant error in the pointing response. It is presumed that the procedure followed would be less susceptible to a possible confound associated with pointing, because the regression line from which the intercept was determined has the potential of averaging out errors in pointing across target locations.)

To evaluate this possibility further, the misrepresentation in eye position necessary to account for the variation in egocentric direction associated with each eye-positionvarying condition was estimated for Subject C.M. The procedure was directly analogous to that used for determining the location of the cyclopean eye necessary to account for the between-eyes difference. For each eyevarying condition, the single proportional over- or underrepresentation of the varying eye's position, expressed in prism diopters, that would best account for the set of observed egocentric directions of the target was estimated. Hering's principles were followed, and a central cyclopean eye was assumed. The set of egocentric directions yielded by this misrepresentation of eye position, when compared with the observed set of egocentric directions by using linear regression analysis, would give a slope of approximately 1 for the best-fit regression line without meaningfully affecting the goodness of fit of the original regression line (shown in Figure 2). For the right-eyevarying condition, an underrepresentation of that eye's position of approximately $25 \%$ was necessary to yield a slope of approximately 1 ; the regression equation was $y$ $=-0.96+1.02 x, r^{2}=0.99$, where $x$ represents the predicted directions based on the $25 \%$ underrepresentation. For the left-eye-varying condition, a $65 \%$ overrepresentation was necessary to yield a set of predicted magnitudes comparable to the set of observed magnitudes; the equation was $y=2.2+1.04 x, r^{2}=.99$. This analysis was also done on the data from the left-eye-varying condition of Subject J.R. and the right-eye-varying condition of Subject D.W., the two other cases in which the slope of the regression line in the original analysis was statistically different from 1 . In these two cases, an over- 
representation of $40 \%$ and an underrepresentation of $15 \%$, respectively, were necessary to yield a slope of 1 .

Does existing evidence support the possibility of such apparently large errors in the sensing of eye position in binocularly normal subjects? The aforementioned study of Mann et al. (1979) reported even larger, relative undersensing of eye position in individuals with a certain type of strabismus, but no errors in normals; however, errors in their normal individuals might have been obscured when the data of the binocularly normal subjects were grouped for reporting and for statistical analysis. And although Walls (1951) theorized that only the position of the dominant eye affected egocentric direction, he provided no quantitative empirical support for this speculation.

Recent data from another sort of experimental paradigm, one in which changes in egocentric direction associated with dynamic changes in eye position were measured, support the possibility of large errors in sensed eye position in visually normal subjects. Honda (1990) had subjects report the extent of change in egocentric visual direction of a target viewed binocularly in the dark by a visually based measure and by open-loop pointing. For both measures, the subjects reported changes in egocentric direction during pursuit eye movements consistent with errors in the sensing of eye position; the visually based measure showed an undersensing of eye position of about $10 \%$ for 2 of the subjects and $20 \%$ for the 3 rd subject, and the pointing measure suggested even larger underrepresentations of up to $50 \%$. (Other data in the oculomotor literature have been interpreted in terms of an underrepresentation of eye position during pursuit; see, e.g., Schlag, Schlag-Rey, \& Dassonville, 1990.) During saccadic eye movements, an overrepresentation of about $5 \%-10 \%$ was evident in the visually based measure, with a similar but smaller and less clear-cut trend in the pointing measure. Thus, a second prediction of unequal weighting of eye position for egocentric direction, one lying outside Hering's principles, seems plausible. Honda's (1990) study, however, like previous studies, did not address the possibility of between-eye differences.

A factor limiting speculation about possible betweeneye differences in the weighting of eye position for egocentric direction is that the specific eye-position information used for egocentric direction in our experimental paradigm is not known but is presumed to be essentially static. Explanation of a between-eyes difference in egocentric direction appears to necessitate a between-eyes difference in the information necessary to position and/or maintain the eye in the required position for each of our eye-position configurations. In our experimental paradigm, this information could have been provided by any or all of a number of factors. These are (1) accommodative vergence, (2) disparity vergence, (3) proximal vergence, and (4) tonic vergence (for an overview, see Schor \& Ciuffreda, 1983). There are various types of models with potential relevance, but none speaks directly to the issue of how each of the various types of vergence information may be related to egocentric direction. At one level, oculomotor models (e.g., Krishnan \& Stark, 1977; Schor \& Kotulak, 1986; Rosenfield \& Gilmartin, 1988) delineate how the eyes achieve a specified position; they do not explicitly address the nature of the information that affects egocentric direction. At another level, "perceptual"' models (e.g., Ono, 1991; Swanston et al., 1990) describe the components contributing to egocentric visual direction, with one of the components being, effectively, information about the vergence posture of the eyes taken as a given. However, whether and how each of the subcomponents of the vergence response contributes to egocentric direction cannot be deduced from these, or from any of the other types of models. At yet another level, models describe the mechanism of visual direction constancy (e.g., Matin, 1986). These define specific sources of information that might provide the visual direction mechanism with information about the posture of the eyes-that is, outflow and/or inflow. In none of the aforementioned models, however, are between-eye differences explicitly modeled.

A sensory aspect? A result consistent with the suggestion of between-eye differences in weight for egocentric direction was provided by Francis and Harwood (1951). They asked subjects to align themselves to two binocularly viewed, distant targets, and found that the subjects positioned themselves so that the distant objects were collinear with a point approximately midway between the two eyes. Francis and Harwood then measured this binocular alignment point when one of the eyes viewed the targets through a neutral density filter. They found that betweeneye differences in luminance caused the alignment to shift toward the unfiltered eye, and that greater reductions in luminance led to greater shifts in alignment in a linear fashion. However, when the filter was switched from one eye to the other, they found that the reduction in luminance in one eye did not have the same effect on egocentric direction as did the reduction in luminance in the other eye in many subjects. Francis and Harwood's data provide a strong suggestion for a between-eyes difference in the weight that each eye carries for egocentric direction, a difference that is related to sensory factors.

Ocular dominance? Francis and Harwood (1951) approached their research in the context of seeking a better understanding of the notion of ocular dominance, a concept that is no less vague today than it was then (cf. Barbeito, 1980, and Porac \& Coren, 1976). It is now known that the dominant eye-if it is operationally defined as the eye preferred for sighting - is highly related to egocentric direction. Barbeito $(1980,1981)$ demonstrated that the sighting-dominant eye and the measured location of the cyclopean eye are usually on the same side of the midline and argued that the eye chosen for sighting is chosen because it is the eye closer to the cyclopean eye. On the basis of similar data, however, Porac and Coren (1986) argued the opposite causal relationship. With the meaning of the dominant eye for individuals-beyond an operational definition-still obscure, to invoke it as an "explanation" for the between-eye differences observed in 
the present study would seem specious. Certainly what is not needed is yet another method for determining the dominant eye. (For annotation and discussion of the myriad methods for determining the dominant eye, see Barbeito, 1980 , or Porac \& Coren, 1976.)

\section{Conclusion}

Resolving the issue of whether between-eye differences observed in the present study are due to a noncentral cyclopean eye or to differential weighting of eye position for egocentric direction is not straightforward. Simply to measure the location of the cyclopean eye directly with one of the procedures specifically designed to do so, and then to compare this location with that necessary to account for the between-eye differences, would not provide a ready answer. For a given subject, the cyclopean eye locations yielded by the various methods proposed to determine empirically its location do not agree. (For a discussion of these procedures and their poor intercorrelations, see Barbeito \& Ono, 1979.) Because each of the cyclopean eye measurement procedures uses visual directionalizations by a subject to estimate the cyclopean eye's location, the visual directionalizations obtained in the present study can also be used to estimate the location of the cyclopean eye, as was done for Subject C.M. The paradigm used in the present study, then, is in effect another method of locating the cyclopean eye. Given what we presently know about the lack of agreement among the cyclopean eye locations yielded by the various measurement procedures, how would agreement, or lack of agreement between (1) the location yielded by any one of the measurement procedures and (2) the location necessary to account for the between-eye differences in directionalizations observed in the present study be interpreted? In light of the question at hand-namely, whether the between-eye differences in variation of visual direction observed in the present study are an indication of a noncentral cyclopean eye or an indication of between-eye differences in the weighting of eye-position information-our present state of knowledge of cyclopean eye measurement does not permit an unequivocal answer using this approach.

Clearly, further research in which the possibility of between-eye differences is considered is necessary to convincingly rule out unequal weighting of eye position as a factor, or, conversely, to establish the viability of the cyclopean eye as an independent concept. But if unequal weighting of eye position is to be rejected in favor of a noncentral cyclopean eye as an explanation of the betweeneye differences we observed, we would still be left with the question of what the cyclopean eye exactly is.

\section{REFERENCES}

BARBEITO, R. (1980). Ocular dominance: An explanation based on sighting behaviour. Unpublished doctoral dissertation, York University, Toronto.

BARBEITO, R. (1981). Sighting dominance: An explanation based on the processing of visual direction in tests of sighting dominance. $\mathrm{Vi}$ sion Research, 21, 855-860.

BARBEITO, R., ONO, H. (1979). Four methods of locating the egocenter: A comparison of their predictive validities and reliabilities. Behavior Research Methods \& Instrumentation, 11, 31-36.

BAuer, J. A., JR., Woods, G. D., \& Held, R. (1969). A device for rapid recording of positioning responses in two dimensions. Behavior Research Methods \& Instrumentation, 1, 157-159.

Bedell, H. E., Klopfenstein, J. F., Yuan, N. (1989). Extraretinal information about eye position during involuntary eye movement: Optokinetic afternystagmus. Perception \& Psychophysics, 46, 579-586.

Bridgeman, B., \& Fishman, R. (1985). Dissociation of corollary discharge from gaze direction does not induce a straight-ahead shift. Perception \& Psychophysics, 37, 523-528.

Francis, J. L., Harwood, K. A. (1951). The variation of the projection centre with differential stimulus and its relation to ocular dominance. In Transactions of the International Optical Congress (pp. 75-87). London: British Optical Association.

GaUthier, G. M., Nommay, D., \& Vercher, J.-L. (1990). The role of ocular muscle proprioception in visual localization of targets. Science, 249, 58-61.

HILL, A. L. (1972). Direction constancy. Perception \& Psychophysics, $11,175-178$

HoNDA, H. (1990). The extraretinal signal from the pursuit-eyemovement system: Its role in the perceptual and the egocentric localization systems. Perception \& Psychophysics, 48, 509-515.

Krishnan, V. V., StaRK, L. (1977). A heuristic model for the human vergence eye movement system. IEEE Transactions on Biomedical Engineering, 24, 44-49.

Mann, V: A., Hein, A., Diamond, R. (1979). Localization of targets by strabismic subjects: Contrasting patterns in constant and alternating suppressors. Perception \& Psychophysics, 25, 29-34.

Matin, L. (1986). Visual localization and eye movements. In K. R. Boff, L. Kaufman, \& J. P. Thomas (Eds.), Handbook of perception and human performance: Vol. 1. Sensory processes and perception (pp. 20-1-20-45). New York: Wiley.

Matin, L., Picoult, E., Stevens, J. K., Edwards, M. W., Young, D., \& MACARTHUR, R. (1982). Oculoparalytic illusion: Visual-field dependent spatial mislocalizations by humans partially paralyzed with curare. Science, 216, 198-201.

Morgan, C. L. (1978). Constancy of egocentric visual direction. Perception \& Psychophysics, 23, 61-68.

ONo, H. (1979). Axiomatic summary and deductions from Hering's principles of visual direction. Perception \& Psychophysics, 25, 473-477.

ONo, H. (1981). On Wells's (1792) law of visual direction. Perception \& Psychophysics, 30, 403-406.

ONo, H. (1991). Binocular visual directions of an object when seen as single or double. In D. Regan (Ed.), Vision and visual dysfunction: Vol. 9. Binocular vision (pp. 1-18). Boca Raton, FL: CRC Press.

Ono, H., \& BARBEITo, R. (1982). The cyclopean eye vs. the sightingdominant eye as the center of visual direction. Perception \& Psychophysics, 32, 201-210.

ONo, H., \&ONDA, G. (1978). Apparent movement, eye movements and phoria when two eyes alternate in viewing a stimulus. Perception, 7, 75-83.

ONo, H., WeBer, E. U. (1981). Nonveridical visual direction produced by monocular viewing. Journal of Experimental Psychology: Human Perception \& Performance, 7, 937-947.

Ono, H., Wilkinson, A., Muter, P., Mitson, L. (1972). Apparent movement and change in perceived location of a stimulus produced by a change in accommodative vergence. Perception \& Psychophysics, 12, $187-192$

PaAp, K. R., Ebenholtz, S. M. (1976). Perceptual consequences of potentiation in the extraocular muscles: An alternative explanation for adaptation to wedge prisms. Joumal of Experimental Psychology: Human Perception \& Performance, 2, 457-468.

Park, K., Shebilske, W. L. (1991). Phoria, Hering's laws, and monocular perception of direction. Joumal of Experimental Psychology: Human Perception \& Pefformance, 17, 219-231.

Porac, C., \& Coren, S. (1976). The dominant eye. Psychological Bulletin, 83, 880-897.

Porac, C., COREN, S. (1986). Sighting dominance and egocentric localization. Vision Research, 26, 1709-1713.

Rosenfield, M., Gilmartin, B. (1988). The effect of vergence adap- 
tation on convergent accommodation. Ophthalmic \& Physiological Optics, 8, 172-177.

SchlaG, J., Schlag-Rey, M., Dassonville, P. (1990). Saccades can be aimed at the spatial location of targets flashed during pursuit. Journal of Neurophysiology, 64, 575-581.

SCHOR, C. M., \& CluffredA, K. J. (1983). Vergence eye movements: Basic and clinical aspects. Boston: Butterworths.

Schor, C. M., \& Kotulak, J. C. (1986). Dynamic interactions between accommodation and convergence are velocity sensitive. Vision Research, 26, 927-942.

Steinbach, M. J., \& SMith, D. R. (1981). Spatial localization after strabismus surgery: Evidence for inflow. Science, 213, 1407-1409.

Swanston, M. T., WADE, N. J., \& ONO, H. (1990). Binocular representation of uniform motion. Perception, 19, 29-34.

ViIRre, E., CADERA, W., \& Vilis, T. (1987). The pattern of changes produced in the saccadic system and vestibuloocular reflex by visually patching one eye. Journal of Neurophysiology, 57, 92-103.

WALLS, G. L. (1951). A theory of ocular dominance. A.M.A. Archives of Ophthalmology, 45, 387-412.

\section{APPENDIX}

At the suggestion of an anonymous reviewer, phorias were measured. This was done in two ways. In the simpler of the methods, the "near" $(40 \mathrm{~cm})$ phoria was determined with an Ophthalmic Telebinocular (Keystone View Co., Meadville, PA). In this method, the subject views a haploscopically visible stimulus - a vertically oriented arrow visible to the left eye, and a horizontally oriented scale visible to the right eye. The arrow is perceived as pointing to a particular value on the scale, according to the magnitude of phoria. For most observers, the arrow drifts about over a relatively narrow range of approximately 1 prism diopter. The data from this method, reported in Table A1 indicate the middle of the range. This measurement represents a true disassociated phoria, but it does not permit the assessment of possible between-eye differences.

The other method of phoria measurement did permit such an assessment. In mimicking the stimulus arrangement of the present study, the phoria of the nonaligned eye associated with each of the eye-position configurations was measured, to the nearest half prism diopter, with a Maddox rod in front of the non-aligned eye. The subject perceived the fixation stimulus in one eye and its vertically streaked image (caused by a horizontal Maddox rod) in the other eye. Subjects were asked to focus the fixation stimulus, as in the experiment. The data reported in Table A1 represent the mean of some 3-5 measurements, depending on variability.

Table A1

Phoria Measurements, Expressed in Prism Diopters, Determined under the Experimental Viewing Conditions with Means and Standard Errors, and with the Keystone Telebinocular (KT)

\begin{tabular}{|c|c|c|c|c|c|c|c|c|c|c|c|c|}
\hline \multirow[b]{3}{*}{ Subject $\dagger$} & \multirow{3}{*}{$\begin{array}{c}\text { Eye } \\
\text { Varying }\end{array}$} & \multicolumn{10}{|c|}{ Experimental Condition* (Fixation Distance, in centimeters) } & \multirow[b]{3}{*}{ KT } \\
\hline & & \multicolumn{2}{|c|}{16} & \multicolumn{2}{|c|}{20} & \multicolumn{2}{|c|}{24} & \multicolumn{2}{|c|}{30} & \multicolumn{2}{|c|}{40} & \\
\hline & & $\boldsymbol{M}$ & $S E$ & $M$ & $S E$ & $M$ & $S E$ & $\boldsymbol{M}$ & $S E$ & $\boldsymbol{M}$ & $S E$ & \\
\hline C.M. & $\begin{array}{l}\text { L.E. } \\
\text { R.E. }\end{array}$ & $\begin{array}{l}3.25 \\
1.20\end{array}$ & $\begin{array}{l}1.7 \\
1.6\end{array}$ & $\begin{array}{l}7.75 \\
1.00\end{array}$ & $\begin{array}{l}1.2 \\
0.4\end{array}$ & $\begin{array}{l}7.00 \\
0.50\end{array}$ & $\begin{array}{l}0.7 \\
0.6\end{array}$ & $\begin{array}{l}3.50 \\
0.20\end{array}$ & $\begin{array}{l}0.5 \\
0.7\end{array}$ & $\begin{array}{l}3.12 \\
0.75\end{array}$ & $\begin{array}{l}0.3 \\
0.5\end{array}$ & 6.5 \\
\hline D.W. & $\begin{array}{l}\text { L.E. } \\
\text { R.E. }\end{array}$ & $\begin{array}{l}28.7 \\
27.3\end{array}$ & $\begin{array}{l}1.3 \\
1.3\end{array}$ & $\begin{array}{l}23.3 \\
25.3\end{array}$ & $\begin{array}{l}1.8 \\
0.9\end{array}$ & $\begin{array}{l}18.0 \\
22.7\end{array}$ & $\begin{array}{l}1.1 \\
1.3\end{array}$ & $\begin{array}{l}10.3 \\
18.7\end{array}$ & $\begin{array}{l}0.9 \\
0.7\end{array}$ & $\begin{array}{r}7.0 \\
12.3\end{array}$ & $\begin{array}{l}0.6 \\
1.4\end{array}$ & 3.0 \\
\hline J.R. & $\begin{array}{l}\text { L.E. } \\
\text { R.E. }\end{array}$ & $\begin{array}{l}-3.7 \\
-4.3\end{array}$ & $\begin{array}{l}0.3 \\
1.2\end{array}$ & $\begin{array}{l}-1.67 \\
-4.67\end{array}$ & $\begin{array}{l}0.3 \\
0.3\end{array}$ & $\begin{array}{l}-1.50 \\
-3.33\end{array}$ & $\begin{array}{l}0.3 \\
0.3\end{array}$ & $\begin{array}{l}-0.50 \\
-1.00\end{array}$ & $\begin{array}{l}0.3 \\
0.6\end{array}$ & $\begin{array}{r}1.00 \\
-0.67\end{array}$ & $\begin{array}{l}0.6 \\
0.3\end{array}$ & 4.5 \\
\hline T.L.S. & $\begin{array}{l}\text { L.E. } \\
\text { R.E. }\end{array}$ & $\begin{array}{l}1.2 \\
0.0\end{array}$ & $\begin{array}{l}0.9 \\
0.0\end{array}$ & $\begin{array}{l}-0.60 \\
-1.20 \\
\end{array}$ & $\begin{array}{l}1.1 \\
0.3\end{array}$ & $\begin{array}{r}0.00 \\
-1.70 \\
\end{array}$ & $\begin{array}{l}0.0 \\
0.5 \\
\end{array}$ & $\begin{array}{l}0.60 \\
0.00 \\
\end{array}$ & $\begin{array}{l}0.5 \\
0.0\end{array}$ & $\begin{array}{l}0.00 \\
0.00\end{array}$ & $\begin{array}{l}0.0 \\
0.0\end{array}$ & 5.0 \\
\hline
\end{tabular}

Note-L.E. = left eye, R.E. = right eye. "Esphoria is represented by positive values. †Subject A.L. was not available for these measurements. 